\title{
The open-object illusion: size perception is greatly influenced by object boundaries
}

\author{
Tal Makovski ${ }^{1}$
}

Published online: 4 May 2017

(C) The Psychonomic Society, Inc. 2017

\begin{abstract}
This study presents a new powerful visual illusion, in which simple "open" objects - ones with missing boundaries-are perceived as bigger than the same size, fully "closed" objects. In a series of experiments that employed a continuous-response adjustment procedure, it was found that the lack of vertical boundaries inflated the perceived width of an object, whereas the lack of horizontal boundaries inflated its perceived length. The effect was highly robust and it was replicated across different stimulus types and experimental parameters, with almost all observers exhibiting a strong effect. In contrast to the overestimation of the size of an object due to missing boundaries, the inclusion of inner boundaries within an object caused observers to underestimate its size, suggesting that filled space sometimes shrinks, rather than inflates, the perceived size of an object. The open-object illusion bears practical implications for graphics and design as well as important theoretical implications. Specifically, it indicates that the perception of an object's area is not veridical but rather critically depends on contour closure. It is suggested that the visual system extends the missing boundaries of open contour objects, which results in an overestimation of the object's size.
\end{abstract}

Keywords Size perception · Visual illusion · Contour closure $\cdot$ Boundary extension $\cdot$ Object perception

Although we are often unaware of it, we estimate the size of an object many times a day, such as when trying to determine whether a car fits into a parking space, or when deciding

Tal Makovski

tal.makovski@gmail.com

1 The Open University of Israel, Ra'anana, Israel whether a box is big enough to hold our stuff. Typically, our visual system performs these estimates efficiently and effortlessly, yet sometimes size perception fails and visual illusions emerge. For instance, it is well established that the context, i.e., the immediate surroundings, plays an important role in size perception (Künnapas, 1955). Consequently, misleading context cues easily distort perception, as demonstrated by the Ebbinghaus and Delboeuf illusions (e.g., Roberts, Harris, \& Yates, 2005), as well as by the Mueller-Lyer illusion, in which the perceived length of a line is modulated by the orientation of inward or outward pointing arrowheads. Given the close relationship between perceived size and distance, there are also numerous examples of how size perception is altered by distance cues (e.g., the Ponzo illusion, Ames room). These illusions showing alleged perceptual failures provide unique opportunities for studying the mechanisms underlying visual perception (Eagleman, 2001). Notably, however, distortions in size perception in the absence of confusing distance or context cues are less common.

Many people have the sense that "open spaces" are bigger than closed ones, or that open windows make the room feels bigger. By contrast, it has also been shown that filled spaces are sometimes perceived as bigger than unfilled spaces, as demonstrated, for example, in the "Oppel-Kundt illusion" (e.g., Mikellidou, \& Thompson, 2014). The role of boundaries in size perception is even less clear when it comes to objects, and thus the present study aimed at investigating how the boundaries of an object and, particularly, the absence of these boundaries, impact its size perception.

Estimating the size of an object often relies on the edges that define the object's shape (Algom, Wolf, \& Bergman, 1985; Stuart, Bossomaier, \& Johnson, 1993). But what if some of the edges are missing? In other words, what is role of contour closure, a key factor in figure-ground segregation and perceptual organization (e.g., Elder \& Zuker, 1998; 
Kovacs \& Julesz, 1993) in size perception? The current experiments addressed this question by testing whether the perceived size of an open contour shape (an object with missing boundaries) differs from the perceived size of a closed contour shape (a fully bounded objet) with the same physical size. The results revealed a strong visual illusion in which simple, "open" objects are perceived as substantially bigger than the same-size, "closed" objects.

\section{Experiment 1}

The first experiment was triggered by an observation that some open objects seem to appear bigger than the same-size closed objects. Using a systematic, continuous-response adjustment procedure, the experiment aimed at confirming this observation and establishing the effect.

\section{Method}

\section{Participants}

All participants were undergraduate students from the Open University of Israel who took part in the experiments for a course credit. All subjects had normal or corrected-to-normal visual acuity. Sixteen participants (four males, age: $M=26.3$ years) participated

\section{Experiment 1}

\section{Equipment and Stimuli}

Participants were tested individually in a dimly lit room. They sat about $67 \mathrm{~cm}$ away from a 17" CRT monitor (resolution $1024 \times 768,85 \mathrm{~Hz}$ ). The experiments were programmed using Psychtoolbox (Brainard, 1997; Pelli, 1997), implemented in MATLAB (http://www.mathworks.com).

Four types of stimuli were tested, two Closed (A \& B) and two Open stimuli (C \& D, Fig. 1, top). Each test stimulus was compared to a probe stimulus, which was a simple rectangle (line thickness: $0.03^{\circ}$ ) presented in one of three sizes: Small: $1.94^{\circ} \times 3.89^{\circ}$, Medium: $3.24^{\circ} \times 6.48^{\circ}$, and Large: $4.54^{\circ} \times$ $9.07^{\circ}$. The initial size of the test stimulus was selected randomly on each trial, with the restriction that its width and length would be within the range of $\pm 1.22^{\circ}$ of the dimensions of the probe.

\section{Procedure and design}

Each trial started with a white cross $\left(1.22^{\circ} \times 1.22^{\circ}\right)$ presented against a blue background for $500 \mathrm{~ms}$. Afterwards, the cross disappeared and a test stimulus appeared together with a probe. The items were presented $2.7^{\circ}$ above or below the center of the screen, and $6.75^{\circ}$ to the left or to the right. Importantly, the two stimuli were presented diagonally to one another to avoid vertical or horizontal alignment (Fig. 1, bottom).

The task of the participants was to adjust the size of the test item so that it would match the size of the probe item. They used the right and left arrow keys to change the width of the test stimulus and the up and down arrow keys to change its length. Accuracy was emphasized and subjects were told to press the space bar to finalize their response only when the size of the two items looked the same.

Each of the four test stimuli appeared with the three probesizes eight times (twice in each of the four positions). This resulted in 96 trials that were presented in a random order.

\section{Results}

The dependent variables were the ratio between the actual size of the probe stimulus (width, length or area, i.e., the multiplication of the two, Algom et al., 1985) and the adjusted size of the test stimulus. Figure 2 plots relative size for each stimulus type and probe-size, separately for width, length and area.

Repeated-measures ANOVAs with stimulus type (A-D) and probe-size (small, medium and large) were performed separately for the relative perceived width, length and area. All of these analyses revealed significant effects of probe-size $\left(p s<.001, \eta_{p}{ }^{2}>.47\right)$, in that the relative perceived size was greater for bigger probes. Importantly, however, there was no interaction between probe-size and stimulus type ( $p \mathrm{~s}>.09)$. No such interaction was also found in the next experiments and therefore the data for probe size were collapsed in the rest of the analyses.

Table 1 shows the mean relative perceived width, length and area of the stimuli tested in Experiment 1. These data reveal that the perceived areas of the "open" stimuli C and D were overestimated by $19 \%$ and $14 \%$, respectively, and this effect was due mainly to the overestimation of the width, rather than length, of the open objects. Accordingly, a repeated-measures ANOVA on relative width found a striking difference between the four stimuli, $F(3,45)=89.19, p<.001$, $\eta_{p}{ }^{2}=.86$, whereby the open objects were estimated as much wider than the closed objects, $F(1,15)=99.87, p<.001$, $\eta_{p}{ }^{2}=.87$. Post-hoc analyses using Bonferroni corrections showed that all pair-wise comparisons were highly significant $(p s<.001)$. There was also a significant difference in length perception, $F(3,45)=14.79, p<.001, \eta_{p}{ }^{2}=.5$. However, this difference was less pronounced, and was driven primarily by stimulus A being judged as shorter than the rest of the objects ( $p$ s $<.001)$, whereas none of the other pair-wise comparisons reached significance. 


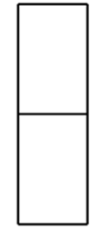

A

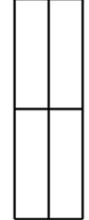

B

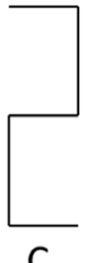

C

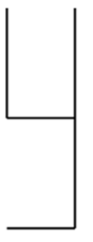

$\mathrm{D}$

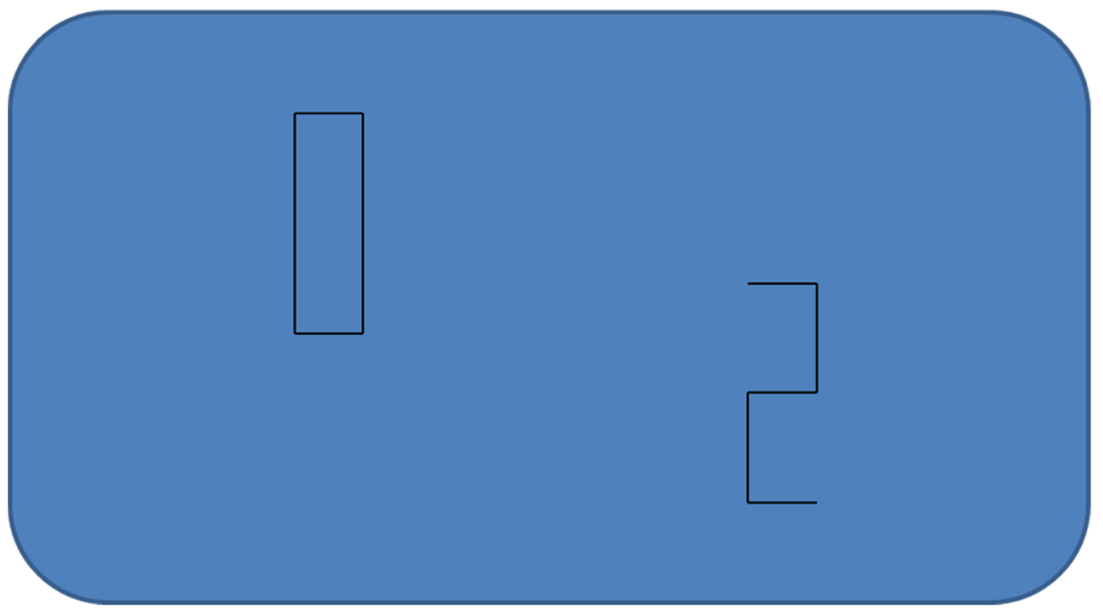

Fig. 1 Top The four stimuli $(A-D)$ tested in Experiment 1. Bottom Example of the stimulus display. Although the physical length and width of the stimuli are identical, the open object (right) is perceived as bigger than the probe (left)

Altogether, these data clearly confirm that the perceived object's area is dramatically influenced by the stimulus type, $F(3,45)=60.32, p<.001$, $\eta_{p}{ }^{2}=.80$, with the open objects being judged as markedly larger than closed objects, $F(1,15)=70.51, p<.001$, $\eta_{p}{ }^{2}=.83$. That is, while there was no difference in the perceived area of stimuli $\mathrm{A}$ and $\mathrm{B}$ and subjects were overall fairly accurate in their size judgments (Saarinen \& Levi, 1999), the open objects C and D were perceived considerably bigger. This pattern was highly consistent with practically all participants showing it, substantiating the conclusion that the effect constitutes a fundamental visual illusion.

\section{Experiment 2}

The goal of Experiment 2 goal was to replicate the findings of Experiment 1 and to test the generality of the effect using slightly different experimental parameters.

\section{Method}

Sixteen new subjects (two males, age: $\mathrm{M}=28.5$ years) were tested in Experiment 2, which repeated the procedure used in Experiment 1 with several modifications. First, the items were presented against a red background and closer to one another. Thus, items were presented $2.16^{\circ}$ above and below the center

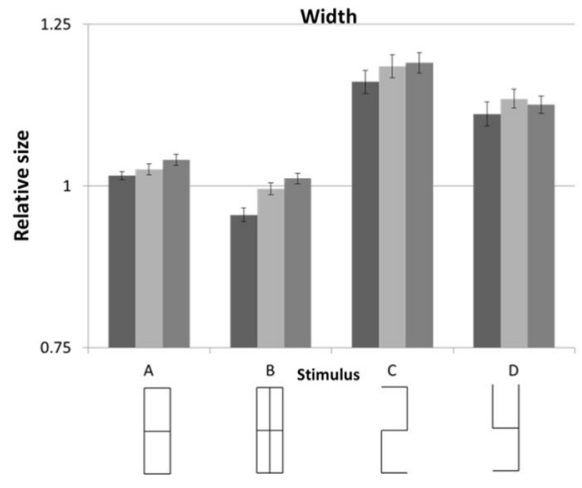

Fig. 2 Results from Experiment 1. Relative width, length and area judgments as a function of stimulus type and probe-size. A value of 1 indicates an accurate stimulus-to-probe match, a value $>1$ an
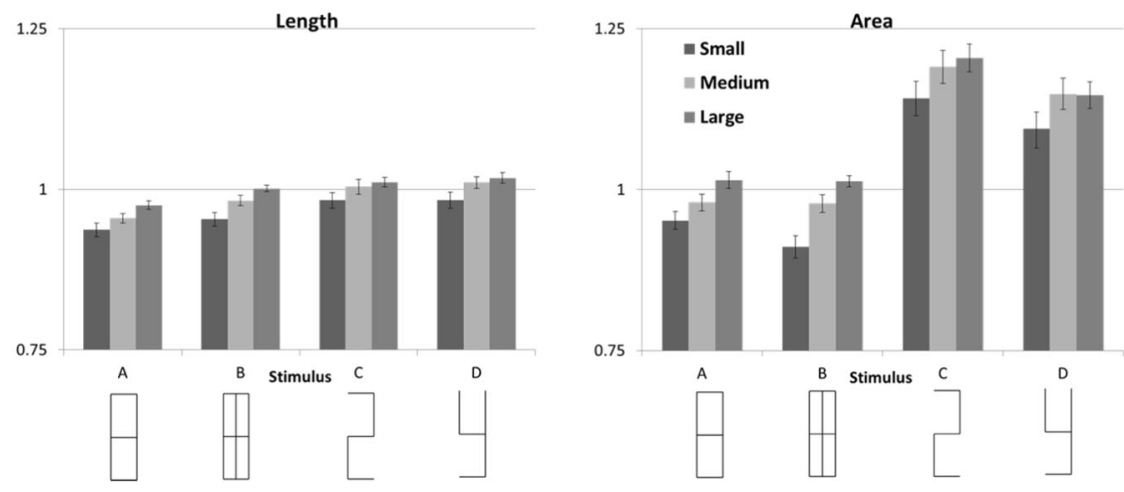

overestimation, and a value $<1$ an underestimation of the adjusted size. Error bars show $\pm 1 \mathrm{SE}$ of the mean 
Table 1 Mean relative size of the stimuli tested in Experiment 1

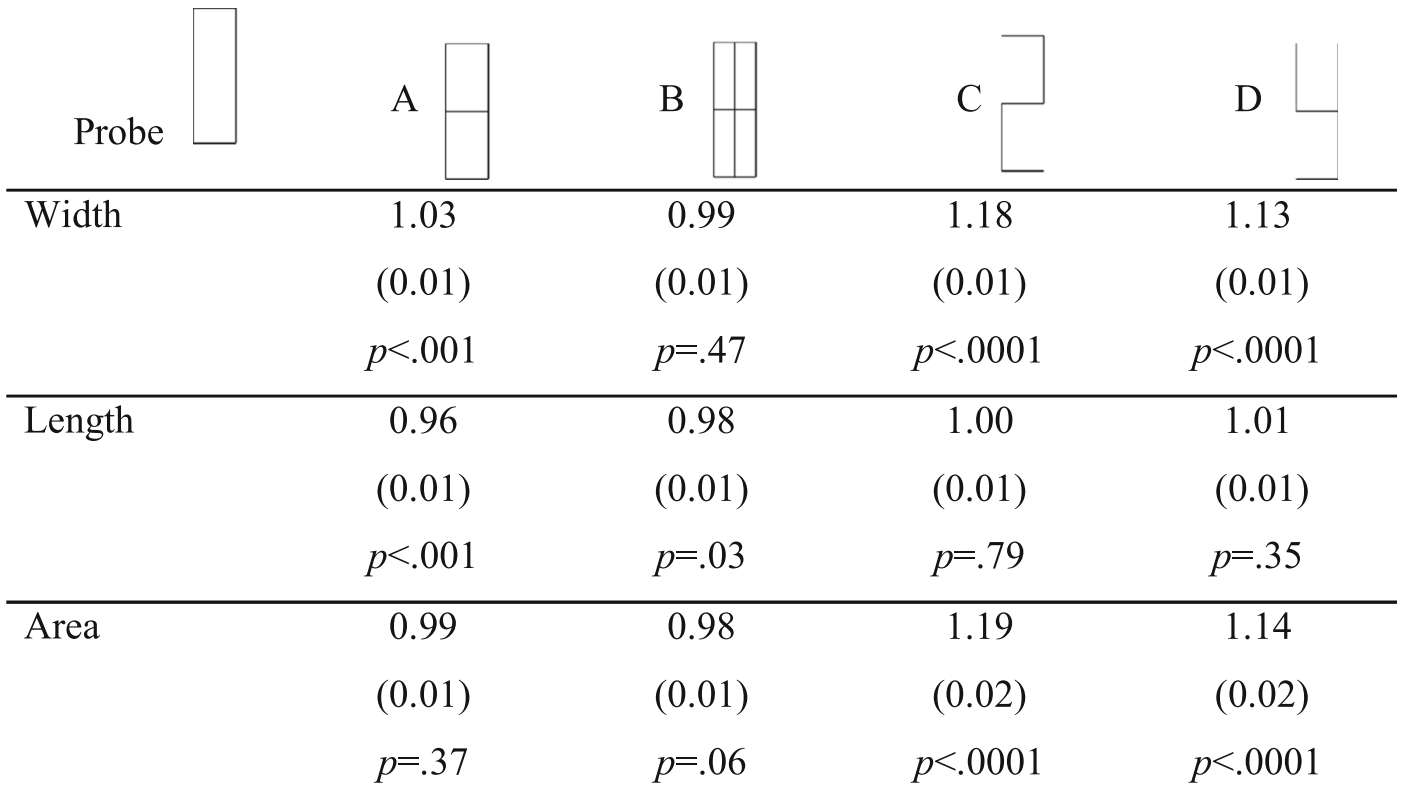

Standard error of the means are presented in parentheses. The $p$-values indicate the results of the $t$-test comparisons to $1(1=$ an accurate match, values $>1$ indicate that the size of the test stimulus was overestimated relative to the probe)

of the screen, and $4.32^{\circ}$ to the left or to the right. Second, three slightly larger probe sizes were used: Small: $2.27^{\circ} \times 4.54^{\circ}$, Medium: $3.78^{\circ} \times 7.56^{\circ}$, and Large: $5.29^{\circ} \times 10.58^{\circ}$.

\section{Results and discussion}

The data from one subject were discarded because he used his hands to gauge the stimuli. Figure 3 depicts the relative width, length and area as a function of stimulus type. The results fully replicated the findings of Experiment 1. There was a highly robust effect of stimulus type on the object's estimated width, $F(3,42)=78.49, p<.001, \eta_{p}{ }^{2}=.85$, area, $F(3,42)=48.36$, $p<.001, \eta_{p}{ }^{2}=.78$, and, to a lesser extent, length, $F(3,42)=$ 5.04, $p=.005, \eta_{p}{ }^{2}=.27$. Importantly, subjects clearly overestimated the area of the open objects (C and D) and judged them as sustainably bigger then the closed objects (A and B), $F(1,14)=65.84, p<.001, \eta_{p}{ }^{2}=.83$. Again, this pattern was observed for all subjects. It is noteworthy that although the objects were presented closer to one another in this experiment compared to Experiment 1, there was no

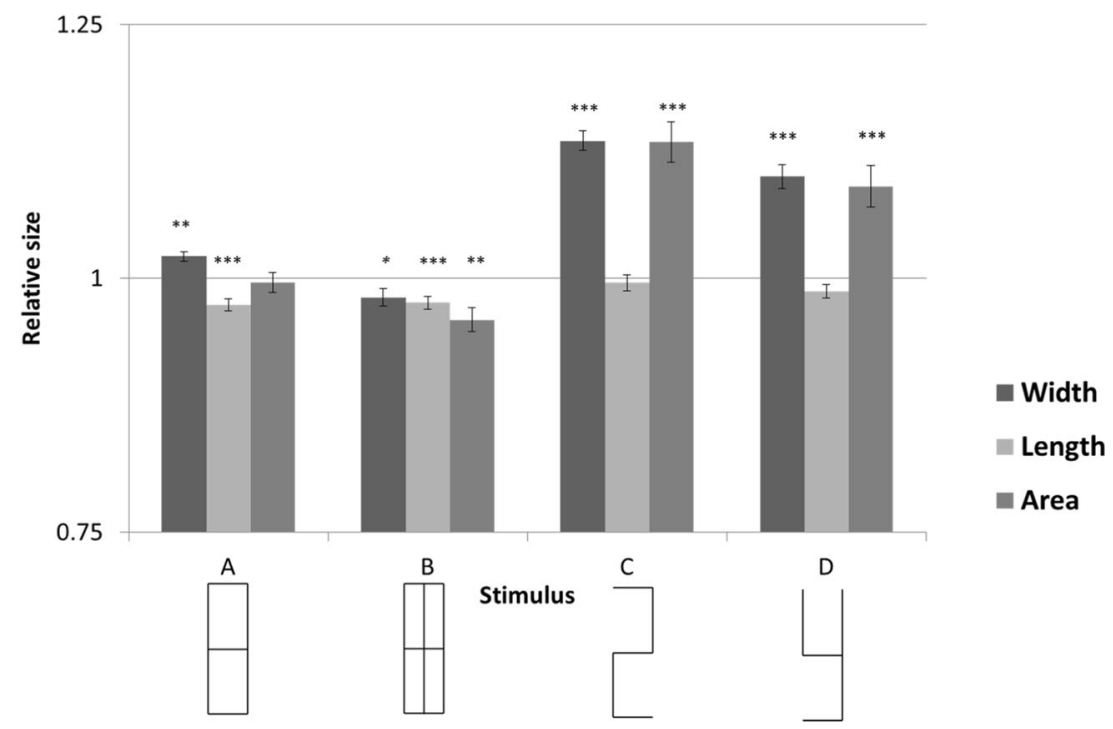

Fig. 3 Results from Experiment 2. Relative width, length and area judgments as a function of stimulus type. Error bars show \pm 1 SE of the mean. ${ }^{*} p$-value of the $t$-test comparison to 1 is $<.05 ; * * p$-values $<.01 ; * * *$-values $<.001$ 
significant difference between the experiments in the perception of visual area, $F(1,29)=2.41, p=.13$, and no interaction between experiment and stimulus type, $F(3,87)=2.12, p=.1$.

\section{Experiment 3}

The goal of Experiment 3 was to test whether the effect is specific to the objects used in Experiments 1 and 2, or can it be extended to other kinds of open objects.

\section{Method}

Fifteen subjects (two males, age: $\mathrm{M}=34.9$ years) were tested with six new open objects (three stimulus types facing left or right, see Fig. 4). The procedure was identical to that used in Experiment 1 except that, because more stimuli were tested, only the medium and large probe sizes were used.

\section{Results and discussion}

Figure 4 depicts the relative width, length and area as a function of stimulus type. Although new open stimuli were used, the results were basically the same. All subjects overestimated the area of the open objects on average by $14 \%$ (range: 6\%$26 \%)$. There was no effect of stimulus type $(E-G)$ or the direction of the open end $(F \mathrm{~s}<1)$. As in the previous experiments, the effect was predominantly due to the overestimation of width, whereas length estimations were fairly accurate. Importantly, these findings confirm the notion that the effect is general and is not limited to few specific objects.

\section{Experiment 4}

Experiments 2 and 3 replicated and extended the finding that size perception is markedly changed when some object boundaries are missing. The effect seems to be driven primarily by the missing vertical boundaries, as the removal of the object's top (stimulus D) or bottom (stimulus G) did not increase the effect. This observation is consistent with the finding that the effect was due mainly to the misperception of the object's width. Nevertheless, the absence of a length effect might be a result of the fact that the missing horizontal boundaries were relatively small. Therefore, the goal of the final experiment was to test whether missing horizontal boundaries can also induce an illusion in which the perceived length, rather than width, is overestimated.

\section{Method}

Sixteen subjects (two males, mean age 27.0 years) participated in Experiment 4. Six types of objects were tested in this experiment, four of them were the rotated versions of the stimuli in Experiment 1 (A-D) and two were new (H-I, Fig. 5). The probe rectangle was also rotated, and only two sizes were used: $6.48^{\circ} \times 3.24^{\circ}$ and $9.07^{\circ} \times 4.54^{\circ}$. In all other respects the experiment was identical to Experiment 1.

\section{Results}

Figure 5 shows the relative size estimates for each of the test stimuli. A repeated-measures ANOVA found that, similar to Experiment 1, the perceived object's area was dramatically influenced by the stimulus type, $F(5,75)=26.31, p<.001$, $\eta_{p}{ }^{2}=.64$. Specifically, there was no difference in the perceived area of the open objects ( $\mathrm{C}$ and $\mathrm{D}, \mathrm{M}=1.08), p=$ .27 , but they were perceived as considerably bigger than the

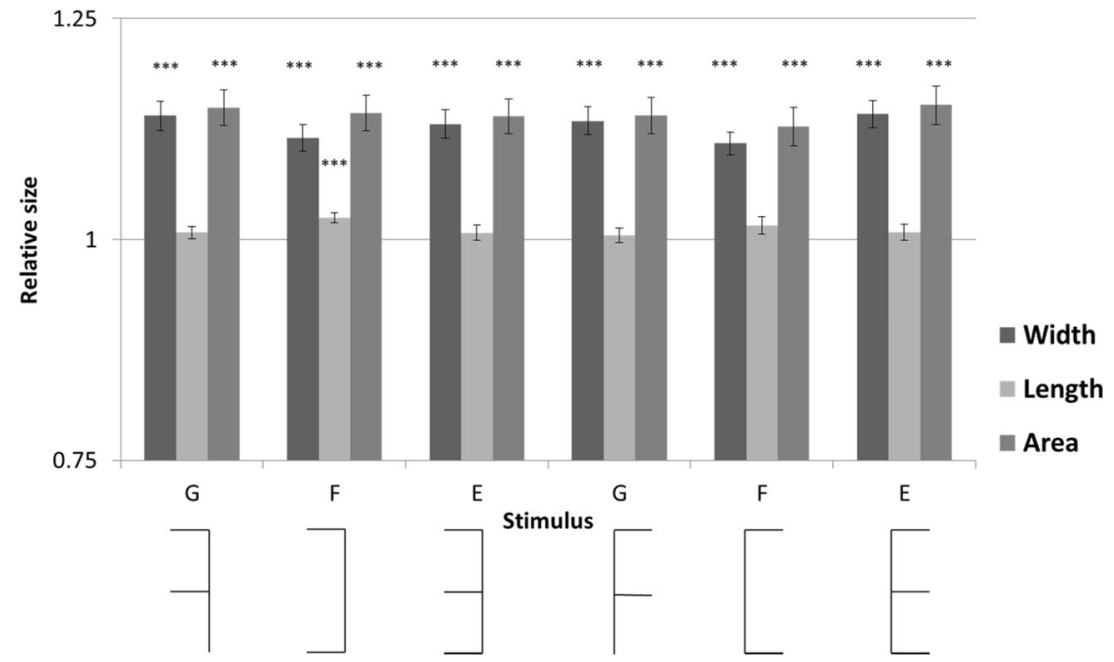

Fig. 4 Results from Experiment 3. Relative width, length and area judgments as a function of stimulus type 


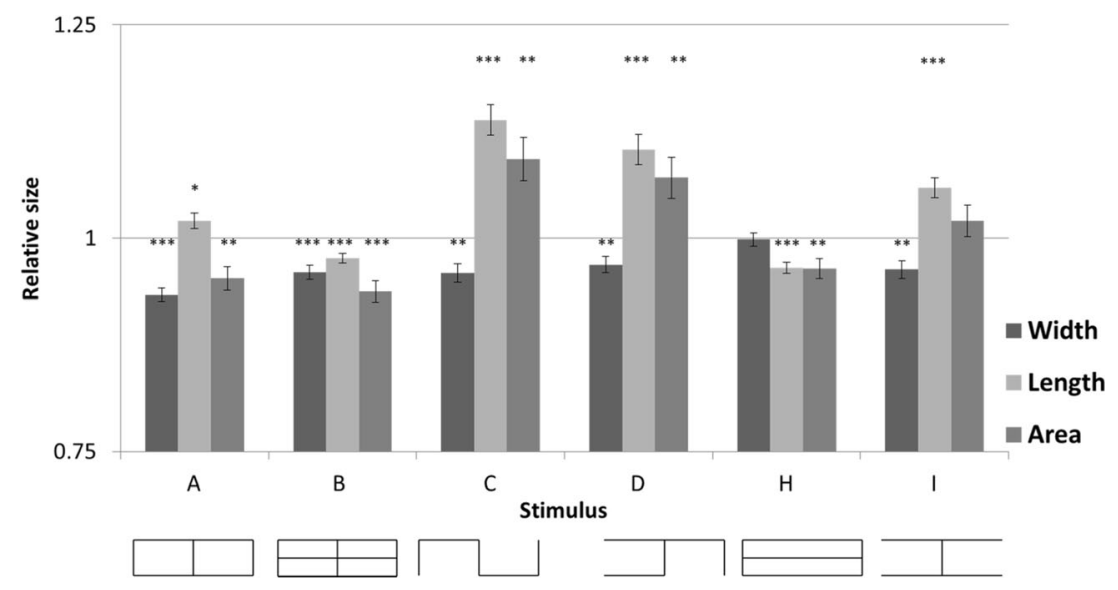

Fig. 5 Results from Experiment 4. Relative width, length and area judgments as a function of stimulus type

closed objects (A, B, H, M = 0.95), $F(1,15)=34.26, p<.001$, $\eta_{p}{ }^{2}=.70$. The effect was diminished for stimulus I that had no horizontal boundary missing, yet it was still perceived bigger than the other closed objects $(p s<.02)$.

Most importantly, the large distortion of the object's perceived area was now driven mainly by the perceived length of the objects, $F(5,75)=51.19, p<.001, \eta_{p}^{2}=.77$. Bonferronicorrected post-hoc analyses confirmed that all pair-wise comparisons were highly significant except for the difference between the two closed objects B and H. In contrast, the difference in width perception was now smaller, $F(5,75)=19.01, p$ $<.001, \eta_{p}{ }^{2}=.56$, with no indication of overestimation (Fig. 5).

\section{Discussion}

Experiment 4 confirmed that the illusion is not confined to object's width. Rather, it is dependent on the side of the missing boundaries, with missing vertical boundaries inflates width perception, and missing horizontal boundaries inflates length perception. Notice, however, that the effect was smaller in the current experiment, probably because of the dominance of the vertical meridian, as illustrated in the vertical-horizontal illusion (Avery \& Day, 1969). Finally, the data showed not only that missing boundaries inflates size perception, but the opposite is also true: the inclusion of inner boundaries shrinks the perceived size of the object, as the closed objects A, B and $\mathrm{E}$ were all underestimated compared to the simple, unfilled probe (similar trends were found in Experiments 1-2, particularly for object B). ${ }^{1}$

\footnotetext{
${ }^{1}$ Underestimation of an object's area when inner boundaries are included, and overestimation of an object's area when some boundaries are missing, were also obtained in a follow-up experiment that used square, rather than rectangular, stimuli. However, the effects were reduced under these conditions, probably because the equal size of the length and width provided a strong cue for making more accurate size judgments.
}

\section{General discussion}

Contour closure is a key element in shape perception (e.g., Murray, Kersten, Olshausen, Schrater, \& Woods, 2002; Saarinen \& Levi, 1999; Wilder, Feldman, \& Singh, 2016) as it is a critical cue for figure-ground organization (e.g., Elder \& Zuker, 1998; Kovacs \& Julesz, 1993). Consequently, it is easier to detect and to learn to recognize closed contour objects than open contour objects (Elder \& Zuker, 1993; Garrigan, 2012), and fully closed objects induce stronger object-based attention effects (Marino \& Scholl, 2005). The present study introduces a new visual illusion indicating that contour closure has also strong implications on size perception. Particularly, open objects with missing boundaries are perceived considerably bigger than closed objects of the same size. This openobject illusion was found to be highly robust and consistent across observers, and was generalized across several stimulus types and experimental parameters. Moreover, there was some evidence that the reversed pattern is also true: the inclusion of inner boundaries makes the object looks smaller. This finding seems in contrast to the "Helmholtz square illusion" or the "Oppel-Kundt illusion", according to which filled spaces appear larger than unfilled spaces (Mikellidou, \& Thompson, 2014; Thompson \& Mikellidou, 2011). However, this underestimation effect was not as large as the open-object illusion, and additional investigation is needed for establishing its robustness.

The open-object illusion is a unique phenomenon in its magnitude, robustness and extent. It can be distinguished from other size perception effects caused by symmetry, meaning, or pronounceability, which tend to be more limited (Jin, Lee, \& Yuan, 2016; New, Doré-Mazars, Cavézian, Pallier, \& Barra, 2016; Reber, et al., 2014; Reber, Wurtz, Knapstad, \& Lervik, 2010). In contrast, the open-object illusion is consistent with Saarinen and Levi's (1999) study showing that shape perception is more accurate for closed contour stimuli than for open contour stimuli. Specifically, Saarinen and Levi found more precise horizontal-vertical ratio discrimination when oriented 
Gabor patches formed a closed, rather than an open, rectangle. The current findings are indeed in agreement with this conclusion, and further raise the possibility that this difference in shape perception might not be driven by the closed stimuli, which their size precision is relatively accurate, but rather by the open stimuli being less accurate, given that their perceived size is largely overestimated.

The open-object illusion bears significant practical implications in various fields related to graphics and design, as it should be taken into account that open and closed visual objects are perceived differently. Furthermore, the illusion has important theoretical implications as it reveals that, even in the absence of context or distance cues, the perception of visual area is not veridical (Wallach \& McKenna, 1960), and depends greatly on object boundaries (Algom et al., 1985; Stuart et al. 1993). But what is the source of the illusion? One explanation would argue that observers perceive the open objects as bigger than they really are because they falsely "extend" the missing boundaries. This tendency to overextend the missing boundaries bears some resemblance to the "occlusion illusion", in which a partly occluded figure is perceived larger than a similarly sized fully visible figure, because both effects seem to somewhat rely on inaccurate partial modal completion (Palmer, Brooks, \& Lai, 2007). Interestingly, this interpretation resonates with the wellknown boundary-extension effect, in which scenes are remembered as having larger boundaries than they actually had (Intraub, \& Richardson, 1989). Yet, in contrast to the typical boundary-extension effect, the open-object illusion seems to suggest that some boundary extension takes place even within perception (Chadwick, Mullally, \& Maguire, 2013), and even in the absence of a scene (Gottesman, \& Intraub, 2002).

Nevertheless, other, not mutually exclusive, explanations of the illusion are also plausible. For instance, it was found that when visual stimuli form a coherent shape, brain activity in higher visual areas is increased, and activity in the primary visual cortex is decreased (Murray et al. 2002). Thus, it is possible that because of the tendency of the visual system to complete the shape of the object, an open contour object involves more feedback from higher visual areas and amplified activity in early visual areas, which in turn results with a larger percept of size. Alternatively, an open object might be more likely to be interpreted by the visual system as a $3 \mathrm{D}$ object slanted away from the observer, and this misinterpretation of depth might have biased size perception. ${ }^{2}$

In sum, this paper presents a new powerful visual illusion in which size perception is inflated for open contour objects. Clearly, many important questions remained to be answered. For example, is the effect unique to objects or it is related to distorted space segmentation (e.g., Mikellidou \& Thompson,

\footnotetext{
${ }^{2}$ I thank an anonymous reviewer for raising these possibilities.
}

2014)? Can the effect be generalized to 3D or curved objects? Is the distinction between open and closed stimuli sharp or gradual? What is the role of the thickness of the boundary in the illusion? More research is therefore needed to fully delineate the boundary conditions of the illusion and its underlying mechanism.

Acknowledgements I thank Ido Liviatan for helpful discussions, and Ayelet Golestani and Tomer Sahar for data collection.

\section{References}

Algom, D., Wolf, Y., \& Bergman, B. (1985). Integration of stimulus dimensions in perception and memory: Composition rules and psychophysical relations. Journal of Experimental Psychology: General, 114(4), 451-471.

Avery, G. C., \& Day, R. H. (1969). Basis of the horizontal-vertical illusion. Journal of Experimental Psychology, 81(2), 376-380.

Brainard, D. H. (1997). The psychophysics toolbox. Spatial Vision, 10(4), 433-436.

Chadwick, M. J., Mullally, S. L., \& Maguire, E. A. (2013). The hippocampus extrapolates beyond the view in scenes: An fMRI study of boundary extension. Cortex, 49(8), 2067-2079.

Eagleman, D. M. (2001). Visual illusions and neurobiology. Nature Reviews Neuroscience, 2, 920-926.

Elder, J., \& Zucker, S. (1993). The effect of contour closure on the rapid discrimination of two-dimensional shapes. Vision Research, 33(7), 981-991.

Elder, J. H., \& Zucker, S. W. (1998). Evidence for boundary-specific grouping. Vision Research, 38, 143-152.

Garrigan, P. (2012). The effect of contour closure on shape recognition. Perception, 41(2), 221-235.

Gottesman, C. V., \& Intraub, H. (2002). Surface construal and the mental representation of scenes. Journal of Experimental Psychology: Human Perception and Performance, 28(3), 589-599.

Intraub, H., \& Richardson, M. (1989). Wide-angle memories of close-up scenes. Journal of Experimental Psychology: Learning, Memory, and Cognition, 15, 179-187.

Jin, Z., Lee, Y., \& Yuan, Z. (2016). Biofunctional understanding and judgment of size. Frontiers in Psychology, 7, 436.

Kovacs, I., \& Julesz, B. (1993). A closed curve is much more than in incomplete one: Closure in figure ground segmentation. Proceedings of the National Academy of Sciences of the Unites States of America, 90, 7495-7497.

Künnapas, T. M. (1955). Influence of frame size on apparent length of a line. Journal of Experimental Psychology, 50(3), 168-170.

Marino, A. C., \& Scholl, B. J. (2005). The role of closure in defining the "objects" of object-based attention. Attention, Perception, \& Psychophysics, 67(7), 1140-1149.

Mikellidou, K., \& Thompson, P. (2014). Crossing the line: Estimations of line length in the Oppel-Kundt illusion. Journal of Vision, 14(8), 20. doi: $10.1167 / 14.8 .20$

Murray, S. O., Kersten, D., Olshausen, B. A., Schrater, P., \& Woods, D. L. (2002). Shape perception reduces activity in human primary visual cortex. Proceedings of the National Academy of Sciences of the United States of America, 99(23), 15164-15169.

New, B., Doré-Mazars, K., Cavézian, C., Pallier, C., \& Barra, J. (2016). The letter height superiority illusion. Psychonomic Bulletin \& Review, 23(1), 291-298.

Palmer, S. E., Brooks, J. L., \& Lai, K. S. (2007). The occlusion illusion: Partial modal completion or apparent distance? Perception, 36, 650 669. 
Pelli, D. G. (1997). The VideoToolbox software for visual psychophysics: Transforming numbers into movies. Spatial Vision, 10(4), 437-442.

Reber, R., Christensen, B. T., \& Meier, B. (2014). Effects of meaning and symmetry on judgments of size. Frontiers in Psychology, 5, 1270.

Reber, R., Wurtz, P., Knapstad, M., \& Lervik, L. V. (2010). Polarity correspondence in comparative number magnitude judgments. Psychonomic Bulletin \& Review, 17(2), 219-223.

Roberts, B., Harris, M. G., \& Yates, T. A. (2005). The roles of inducer size and distance in the Ebbinghaus illusion (Titchener circles). Perception, 34(7), 847-856.

Saarinen, J., \& Levi, D. M. (1999). The effect of contour closure on shape perception. Spatial Vision, 12, 227-238.
Stuart, G. W., Bossomaier, T. R., \& Johnson, S. (1993). Preattentive processing of object size: Implications for theories of size perception. Perception, 22(10), 1175-1193.

Thompson, P., Mikellidou, K., \& 1. (2011). Applying the Helmholtz illusion to fashion: Horizontal stripes won't make you look fatter. iPerception, 2, 69-76.

Wallach, H., \& McKenna, V. V. (1960). On size-perception in the absence of cues for distance. The American Journal of Psychology, 73(3), 458-460.

Wilder, J., Feldman, J., \& Singh, M. (2016). The role of shape complexity in the detection of closed contours. Vision Research, 126, 220-231. 